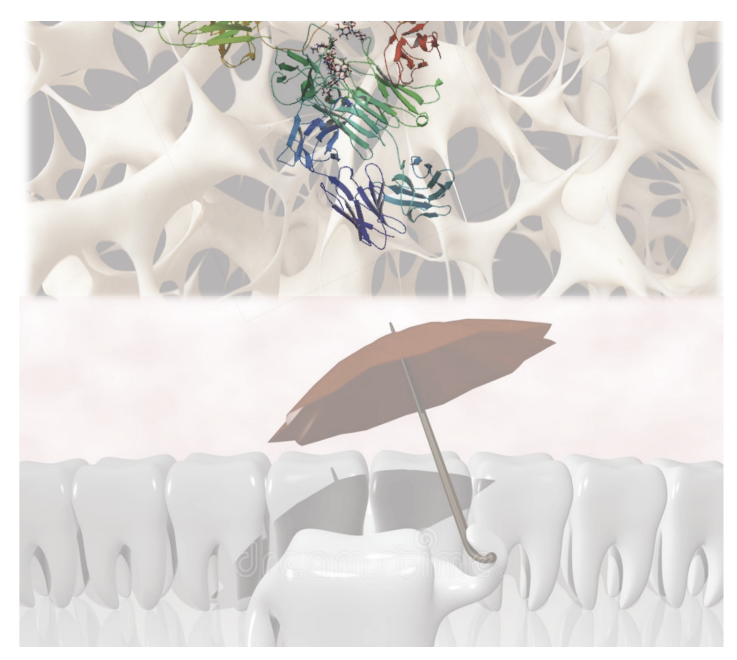

\title{
Simplifying the dental/periodontal management of patients with metabolic bone fragility receiving treatment with denosumab
}

Giuseppina Campisi ${ }^{1}$, Rodolfo Mauceri ${ }^{1}$, Francesco BERT OLDO², Vittorio Fusco ${ }^{3}$, Alberto

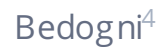

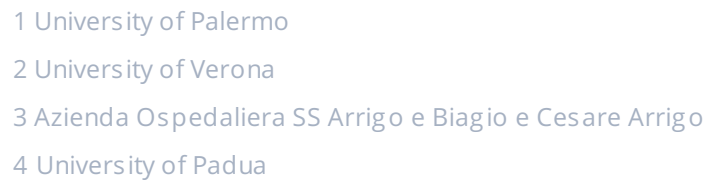

Funding: The author(s) received no specific funding for this work.

Potential competing interests: Giuseppina Campisi, Vittorio Fusco and Rodolfo Mauceri have nothing to disclose. Francesco Bertoldo serves as Advisory board for Amgen, UCB, SPA, Janssen Pharmaceutica, Astellas Pharma. He also received honoraria and/or travelling grants for lecturing. Alberto Bedogni received honoraria and/or travelling grants for lecturing from Amgen.

\section{Abstract}

Denosumab (DNB) is a bone-targeted medication used to preserve structural integ rity and minimise the risk of frag ility fractures in metastatic cancer and metabolic bone disorders. DNB targets and binds RANK Ligand, inhibiting osteoclast maturation, function, and survival. In contrast with nitrogen-containing bisphosphonates (N-BPs), DNB does not bind to hydroxyapatite and incorporate into bone; thus, bone cellular remodelling recovers rapidly after drug suspension. 
Denosumab has benn linked to the occurrence of osteonecrosis of the jaw (MRONJ), a uncommon but severe oral side effect with a higher prevalence in metastatic cancer patients than in patients with metabolic bone frag ility. Althoug heveral oral triggers can initiate MRONJ, invasive oral treatments and tooth extraction still remain the most common precipitating event. In general, tooth extraction and oral surgery should be avoided in patients at increased risk of MRONJ, while extraction of unsalvageable teeth should be performed based on specific risk reduction protocols to eliminate dental/periodontal infections, still protectig from MRONJ onset. Based on the different pharmacological properties of DNB and N-BPs, it is likely that the MRONJ risk profile of patients with metabolic bone frag ility receiving receiving different ARs could somewhat vary. We hypothesize the chance to maximize the pharmacokinetic of Prolia $₫$ and identify a time interval in which invasive oral treatments can ideally take place without restrictions in patients with metabolic bone fragility, provided that careful case selection, adequate communication among specialists, planning of a delayed dosing window and rigorous postoperative follow-up are granted.

\section{Definitions}

Medication related osteonecrosis of the jaw (MRONJ)

Defined by Alberto Bedog ni et al.

Risk Factor

Defined by National Cancer Ins titute

Metabolic Bone Disorder

Defined by National Cancer Institute

Risk Assessment

Defined by National Cancer Ins titute

Dosage Regimen

Defined by National Cancer Ins titute

Prognosis

Defined by National Cancer Ins titute

\section{Osteoclast}

Defined by National Cancer Ins titute

\section{Prevalence}

Defined by National Cancer Institute

Multiple myeloma

Defined by INSERM

Secondary Prevention

Defined by National Cancer Institute 
Skeletal-related event (SRE)

Defined by Robert E. Coleman

Cancer Treatment Induced Bone Loss (CTIBL)

Defined by Laura Boehnke Michaud et al.

FDA definition of Scheletal related event (SRE)

Defined by Food and Drug Administration (FDA)

Anti-Angiogenic Drugs

Defined by Han-Chung Wu

In recent years, a new antiresorptive drug (AR) called denosumab (DNB) has been approved worldwide for use in patients with cancer or metabolic bone disorders. DNB targets and binds RANK Ligand, inhibiting osteoclast maturation, function, and survival. [1] [2] In contrast with nitrogen-containing bisphosphonates (N-BPs), DNB does not bind to hydroxyapatite and incorporate into bone; thus, bone cellular remodelling recovers rapidly after drug suspension, with a rebound of bone turnover. ${ }^{[3]}$

Denosumab proved to perform better than zoledronic acid and other N-BPs in terms of prevention of skeletal related event (SRE) in patients with bone metastases ${ }^{[4]}$ and is a valid alternative to bisphosphonates for the reduction of fracture risk in osteoporosis. ${ }^{[5]}$ Different formulations and dosages of DNB are recommended for prevention of SRES in metastatic cancer patients and myeloma patients (Xgeva ${ }^{\circledR}, 20 \mathrm{mg}$ SC q4 weeks) and for prevention of fragility fractures in high risk patients (Prolia ${ }^{\circledR}, 60 \mathrm{mg}$ SC q6 months). At present, Prolia ${ }^{\circledR}$ is indicated for several osteometabolic disorders including: 1) treatment of osteoporosis in postmenopausal women and in men at increased risk of fractures, 2) cancer treatment induced bone loss (CTIBL) associated with hormone ablation in men and women with non-metastatic prostate and breast cancer respectively, who are at increased risk of frag ility fractures and, 3) treatment of bone loss associated with long-term systemic glucocorticoid therapy in adult patients at increased risk of fracture ${ }^{[6]}$.

Based on the reported advantages of the use of DNB over N-BPs, ${ }^{[5]}[7][8]$ there has been a progressive increase in the number of DNB prescriptions worldwide, while prescriptions for bisphosphonates and other osteoporosis medications decreased in many countries. [9] [10]

\section{Medication Related OsteoNecrosis of the Jaw (MRONJ)}

MRONJ is an adverse drug reaction described as the progressive destruction and death of bone that affects the mandible and maxilla of patients exposed to the treatment with medications known to increase the risk of disease, in the absence of a previous radiation 
treatment. ${ }^{[11]}$

N-BPs and DNB have been associated to MRONJ onset [12] [13], alone or in combination with antiang iogenic (AA) drugs . [14]

The prevalence of MRONJ in patients with osteometabolic disorders ranges between $0 \%$ and $0.4 \%$, and it is definitely much less than observed in metastatic cancer patients (between $0.2 \%$ and $6.7 \%$ ). ${ }^{[12]}$

In addition, MRONJ clinical course in patients with metabolic bone disorders is seemingly less severe than usually seen in cancer and myeloma patients receiving high-dose ARs. [13] [15]

\section{MRONJ risk factors and "triggers"}

Despite MRONJ occurrence has been linked to several risk factors, including 1) the given AR medication (i.e. type and dosage schedule), 2) the patient disease profile (bone metastases and metabolic bone fragility), 3) the ongoing cancer therapy, 4) the chronic use of immunosuppressant drugs and steroids and 5) the associated comorbidities, ${ }^{[12]}$ [16] it is still unknown what factor is most likely to impact on disease course and prognosis.

Several oral triggers apparently can initiate MRONJ, including dental and periodontal infection, ill-fitting dentures and dental extraction. Despite a growing body of evidence suggests that dental infection might represent the main local risk factor for MRONJ, [17] tooth extraction still remains the most common precipitating event, accounting for up to 2/3 of the reported MRONJ cases. [12] [17] [13]

In contrast, several studies has proved that surgical tooth extraction, including alveoloplasty and primary wound closure is very successful and protects high-risk patients from MRONJ development. [18] [19] Since then, several risk reduction strategies implemented the routine use of simple and surgical extraction of unsalvageable teeth to eliminate dental/periodontal infections and minimize the risk of MRONJ onset in patients undergoing AR treatment (secondary prevention ). [20]

Assessment of individual MRONJ risk profile (high risk vs low risk) becomes critical to select the appropriate dental treatment and protect patients from unnecessary (overtreatment) or insufficient (undertreatment) interventions.

The cumulative risk of MRONJ in patients receiving ARs for bone metastasis and metabolic bone fragility increases with the time and varies based on the rate of bone turnover suppression that largely depends on the dosage regimen and the duration of treatment; that risk is at least comparable for N-BPs and DNB. [21]

Cumulative dosage (i.e. dose $x$ number of given doses) plays a key role in the individual risk assessment of MRONJ due to N-BPs, irrespective of the route of administration, but 
not necessarily for patients receiving DNB, as it does not incorporate into bone. ${ }^{[17]}$ Based on the different pharmacological properties of DNB and N-BPs, it is likely that the MRONJ risk profile of patients at increased risk of fragility fractures receiving different ARs could somewhat vary. As a consequence, it is rational to stratify the individual risk of MRONJ in patients with metabolic bone fragility also based on the type of drug received as described in Figure 1.

\begin{tabular}{|l|l|l|}
\hline $\mathbf{R}_{\mathbf{0}}$ & $\begin{array}{l}\text { patients* eligible and not yet treated with } \mathbf{A R} \text { medication } \\
\text { patients* exposed to AR medication for less than } 3 \text { years, in the } \\
\text { absence of other systemic or local risk factors (i.e. concomitant use of } \\
\text { corticosteroids, diabetes, rheumatoid arthritis) }\end{array}$ \\
$\mathbf{R}_{\mathbf{X}}$ & $\begin{array}{l}\text { patients* exposed to AR medication for more than } 3 \text { years } \\
\text { patients* exposed to AR for less than } 3 \text { years but in the presence of } \\
\text { other systemic or local risk factors }\end{array}$ \\
\hline
\end{tabular}

* it includes also CTIBL patients (Hormone deprivation therapy induced bone loss in breast and prostate cancer patients without bone metastases)

Figure 1: MRONJ risk profile of patients with metabolic bone fragility receiving AR medications

Patients at increased risk of fragility fractures who are shifted from NBP to DNB treatment represent a separate group where the cumulative dosage of the NBP leads the individual risk of MRONJ occurrence. ${ }^{[22]}$

\section{Pharmacokinetic of denosumab.}

After sc. administration, serum concentrations of DNB ${ }^{\circledR}$ (fl. sc. 60mg) peaks at around day 10 and level to pre-dose values at 24-26 weeks. This has been observed after single and multiple injections in different racial groups and body weight[. [23] [24] After a single dose of Prolia ${ }^{\circledR}$, bone resorption markers (serum CTX levels) reach nadir within 3-7 days and decreasing by up to $80 \%$ from baseline levels. Bone turnover remains suppressed for at least 150 days after last administration [24] [25] and gradually 
regains the pre-dose values within 7-8 months (1-2 month off-therapy). [23] [24] [26]

Treatment interruption leads to reversal of the Prolia ${ }^{\circledR}$ effect on bone mineral density (BMD) to pretherapy levels within 1 year. ${ }^{[3]}$

In addition, stopping Prolia ${ }^{\circledR}$ in patients at increased risk of fragility fractures has been associated with a rebound vertebral fracture risk. ${ }^{[1]}$ [27] $^{[27}$ For this reason, a drug-holiday prior to surgical dental treatment is not advisable at present.

Nevertheless, it is still possible to maximize the pharmacokinetic of Prolia ${ }^{\circledR}$ and identify a time interval in those postponable and noncritical dental/periodontal conditions requiring invasive treatment can ideally take place without restrictions. This "delayed dosing window" lasts about 2 months, starts ideally 5 months after the last dose of Prolia $\circledR$ and ends at the beginning of the 7th month. Over such timespan, bone remodelling is likely to occur and stimulate bone and soft-tissue healing following invasive dental treatments, similar to naïve patients. On the other hand, 1-month postponement of Prolia $₫$ would not compromise bone mineral density, still protecting patients from an increased fracturative risk. (Figure 2)

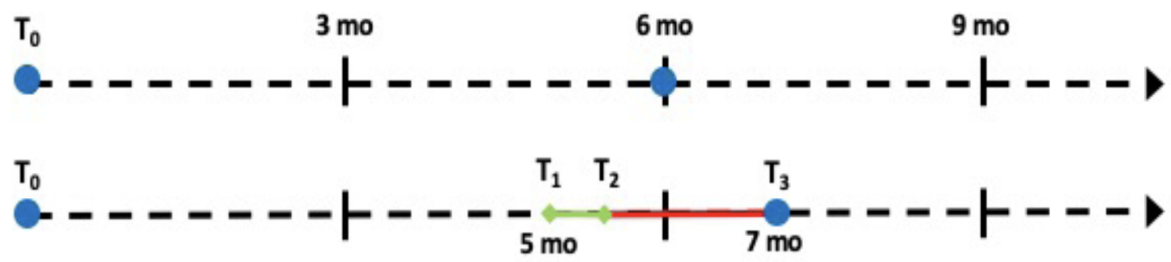

- administration of Prolia ${ }^{\circledR}$ TO : last administration of Prolia ${ }^{\circledR}$ T1 -T2 : 15 days of therapeutic window to perform dental surgical procedure, planned 5 months after last administration (the therapeutic window must be agreed with the prescriber) T2 -T3: healing period (4-6 weeks) T3: resumption of Prolia ${ }^{\circledR}, 7$ months after last administration and, in any case, at least 4 weeks after the last invasive dental procedure

Figure: "Delayed dosing window" of Prolia ${ }^{\circledR}$ and timing of elective oral and dentoalveolar surgery in patients with metabolic bone fragility

Invasive oral treatment of non emergent dental/periodontal conditions in patients receiving Prolia®. 
The chance to adopt a "delayed dosing window" to perform unrestricted elective oral and dentoalveolar surgery depends on the ability of the dental practitioner to:

1. identify the dental/periodontal conditions whom treatment can be reasonably postponed to the 5th month from the last denosumab injection (i.e elective dentoalveolar and periodontal surgery, nonurgent tooth extraction), from those who require urgent management; these latter should not be delayed in any case and finalized according to well-suited risk reduction strategies that represent an effective means of reducing the incidence of MRONJ associated with ARs; [20]

2. directly interact with the bone specialist (drug prescriber), communicate the treatment plan and profile the appropriate "delayed dosing window". Then, truly provide patients with exhaustive information about the possible risk and benefit of the planned procedure;

3. treat the patient according to routine dental protocols and strictly follow-up the healing process;

4. promptly communicate the progress of healing to the bone specialist, who will jointly evaluate the opportunity to restart DNB.

In conclusion, we hypothesize that invasive oral treatment of non emergent dental/periodontal conditions can be performed without restrictions in patients with metabolic bone frag ility receiving Prolia ${ }^{\circledR}$, provided that careful case selection, adequate communication among specialists, planning of a delayed dosing window and rigorous postoperative follow-up are granted. Longitudinal clinical studies are needed to endorse its adoption in the dental practice.

\section{References}

1. a, b Henry G Bone, Rachel B Wagman, Maria L Brandi, Jacques P Brown, Roland Chapurlat, Steven R Cummings. (2017). 10 years of denosumab treatment in postmenopausal women with osteoporosis: results from the phase 3 randomised FREEDOM trial and open-label extension. The Lancet Diabetes \& Endocrinology, vol. 5 (7), 513-523. doi:10.1016/s2213-8587(17)30138-9.

2. ^Sarah Zaheer, Meryl LeBoff, E Michael Lewiecki. (2015). Denosumab for the treatment of osteoporosis. Expert Opinion on Drug Metabolism \& Toxicology, vol. 11 (3), 461-470. doi:10.1517/17425255.2015.1000860.

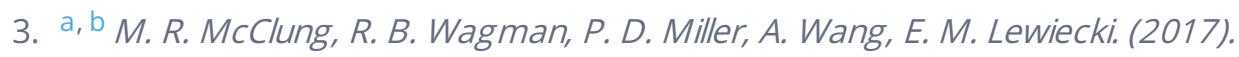


Observations following discontinuation of long-term denosumab therapy. Osteoporos Int, vol. 28 (5), 1723-1732. doi:10.1007/s00198-017-3919-1.

4. `A. Lipton, K. Fizazi, A.T. Stopeck, D.H. Henry, M.R. Smith, N. Shore. (2016). Effect of denosumab versus zoledronic acid in preventing skeletal-related events in patients with bone metastases by baseline characteristics. European Journal of Cancer, vol. 53, 75-83. doi:10.1016/j.ejca.2015.09.011.

5. a, b Houchen Lyu, Bakr Jundi, Chang Xu, Sara K Tedeschi, Kazuki Yoshida, Sizheng Zhao. (2018). Comparison of Denosumab and Bisphosphonates in Patients With Osteoporosis: A Meta-Analysis of Randomized Controlled Trials. doi:10.1210/jc.201802236.

6. ^ EMA - European Medicines Agency.. Prolia. Eur Public Assess Rep 2010.

7. `Jacques P Brown, Richard L Prince, Chad Deal, Robert R Recker, Douglas P Kiel, Luiz H de Gregorio. (2009). Comparison of the Effect of Denosumab and Alendronate on BMD and Biochemical Markers of Bone Turnover in Postmenopausal Women With Low Bone Mass: A Randomized, Blinded, Phase 3 Trial*. Journal of Bone and Mineral Research, vol. 24 (1), 153-161. doi:10.1359/jbmr.0809010.

8. ^ L. Karlsson, J. Lundkvist, E. Psachoulia, M. Intorcia, O. Ström. (2015). Persistence with denosumab and persistence with oral bisphosphonates for the treatment of postmenopausal osteoporosis: a retrospective, observational study, and a metaanalysis. Osteoporos Int, vol. 26 (10), 2401-2411. doi:10.1007/s00198-015-3253-4.

9. ^ Pradnya Naik-Panvelkar, Sarah Norman, Zain Elgebaly, Jeff Elliott, Allan Pollack, Jill Thistlethwaite. (2020). Osteoporosis management in Australian general practice: an analysis of current osteoporosis treatment patterns and gaps in practice. BMC Fam Pract, vol. 21 (1). doi:10.1186/s12875-020-01103-2.

10. ^Anju Parthan, Morgan Kruse, Nicole Yurgin, Joice Huang, Hema N. Viswanathan, Douglas Taylor. (2013). Cost Effectiveness of Denosumab versus Oral Bisphosphonates for Postmenopausal Osteoporosis in the US. Appl Health Econ Health Policy, vol. 11 (5), 485-497. doi:10.1007/s40258-013-0047-8.

11. `Alberto Bedogni, Giuseppina Campisi, Vittorio Fusco. (2018). Medication related osteonecrosis of the jaw (MRON/). doi:10.32388/594095.

12. a, b, c, d Salvatore L. Ruggiero, Thomas B. Dodson, John Fantasia, Reginald Goodday, Tara Aghaloo, Bhoomi Mehrotra. (2014). American Association of Oral and Maxillofacial Surgeons Position Paper on Medication-Related Osteonecrosis of the Jaw -2014 Update. Journal of Oral and Maxillofacial Surgery, vol. 72 (10), 1938-1956. doi:10.1016/j.joms.2014.04.031.

13. a, b, c Ourania Nicolatou-Galitis, Morten Schiødt, Rui Amaral Mendes, Carla Ripamonti, Sally Hope, Lawrence Drudge-Coates. (2019). Medication-related osteonecrosis of the 
jaw: definition and best practice for prevention, diagnosis, and treatment. Oral Surgery, Oral Medicine, Oral Pathology and Oral Radiology, vol. 127 (2), 117-135. doi:10.1016/j.00oo.2018.09.008.

14. ` Ourania Nicolatou-Galitis, for the MASCC Bone Study Group, Maria Kouri, Erofili Papadopoulou, Emmanouil Vardas, Dimitra Galiti. (2018). Osteonecrosis of the jaw related to non-antiresorptive medications: a systematic review. Support Care Cancer, vol. 27 (2), 383-394. doi:10.1007/s00520-018-4501-x.

15. ^ F. Saad, J.E. Brown, C. Van Poznak, T. Ibrahim, S.M. Stemmer, A.T. Stopeck. (2012). Incidence, risk factors, and outcomes of osteonecrosis of the jaw: integrated analysis from three blinded active-controlled phase III trials in cancer patients with bone metastases. Annals of Oncology, vol. 23 (5), 1341-1347. doi:10.1093/annonc/mdr435.

16. ^ Giuseppina Campisi, Stefano Fedele, Vittorio Fusco, Giuseppe Pizzo, Olga Di Fede, Alberto Bedogni. (2014). Epidemiology, clinical manifestations, risk reduction and treatment strategies of jaw osteonecrosis in cancer patients exposed to antiresorptive agents. Future Oncology, vol. 10 (2), 257-275. doi:10.2217/fon.13.211.

17. a, b, c Morten Schiodt, Sven Otto, Stefano Fedele, Alberto Bedogni, Ourania Nicolatour Galitis, Roman Guggenberger. (2019). Workshop of European task force on medication-related osteonecrosis of the jaw-Current challenges. Oral Dis, vol. 25 (7), 1815-1821. doi:10.1111/odi.13160.

18. ^ Giorgia Saia, Stella Blandamura, Giordana Bettini, Anita Tronchet, Andrea Totola, Giorgio Bedogni. (2010). Occurrence of Bisphosphonate-Related Osteonecrosis of the Jaw After Surgical Tooth Extraction. Journal of Oral and Maxillofacial Surgery, vol. 68 (4), 797-804. dol:10.1016/j.joms.2009.10.026.

19. ^ Sven Otto, Matthias Tröltzsch, Vesna Jambrovic, Sappasith Panya, Florian Probst, Oliver Ristow. (2015). Tooth extraction in patients receiving oral or intravenous bisphosphonate administration: A trigger for BRONJ development?. Journal of CranioMaxillofacial Surgery, vol. 43 (6), 847-854. doi:10.1016/j.jcms.2015.03.039.

20. a, b Olga DiFede, Vera Panzarella, Rodolfo Mauceri, Vittorio Fusco, Alberto Bedogni, Lorenzo Lo Muzio. (2018). The Dental Management of Patients at Risk of MedicationRelated Osteonecrosis of the Jaw: New Paradigm of Primary Prevention. BioMed Research International, vol. 2018, 1-10. doi:10.1155/2018/2684924.

21. ^ Sven Otto, Christoph Pautke, Tim Van den Wyngaert, Daniela Niepel, Morten Schiødt. (2018). Medication-related osteonecrosis of the jaw: Prevention, diagnosis and management in patients with cancer and bone metastases. Cancer Treatment Reviews, vol. 69, 177-187. doi:10.1016/j.ctrv.2018.06.007.

22. ^ B. Uebelhart, R. Rizzoli, S. L. Ferrari. (2017). Retrospective evaluation of serum CTX levels after denosumab discontinuation in patients with or without prior exposure to 
bisphosphonates. Osteoporos Int, vol. 28 (9), 2701-2705. doi:10.1007/s00198-0174080-6.

23. a, b Henry G. Bone, MichaelA. Bolognese, Chui Kin Yuen, David L. Kendler, Paul D. Miller, Yu-Ching Yang. (2011). Effects of Denosumab Treatment and Discontinuation on Bone Mineral Density and Bone Turnover Markers in Postmenopausal Women with Low Bone Mass. The Journal of Clinical Endocrinology \& Metabolism, vol. 96 (4), 972 980. doi:10.1210/jc.2010-1502.

24. a, b, c Qian Chen, Chaoying Hu, Yanmei Liu, Rong Song, Wenjing Zhu, Hongxin Zhao. (2018). Pharmacokinetics, pharmacodynamics, safety, and tolerability of single-dose denosumab in healthy Chinese volunteers: A randomized, single-blind, placebocontrolled study. PLOS ONE, vol. 13 (6), e0197984. doi:10.1371/journal.pone.0197984.

25. ^Steven R Cummings, Serge Ferrari, Richard Eastell, Nige/ Gilchrist, Jens-Erik Beck Jensen, Michael McClung. (2017). Vertebral Fractures After Discontinuation of Denosumab: A Post Hoc Analysis of the Randomized Placebo-Controlled FREEDOM Trial and Its Extension.J Bone Miner Res, vol. 33 (2), 190-198. doi:10.1002/jbmr.3337.

26. ^ Michael R. McClung, E. Michael Lewiecki, Stanley B. Cohen, Michael A. Bolognese, Grattan C. Woodson, Alfred H. Moffett. (2006). Denosumab in Postmenopausal Women with Low Bone Mineral Density. N EnglJ Med, vol. 354 (8), 821-831. doi:10.1056/nejmoa044459.

27. `Athanasios D Anastasilakis, Stergios A Polyzos, Polyzois Makras, Berengere AubryRozier, Stella Kaouri, Olivier Lamy. (2017). Clinical Features of 24 Patients With Rebound-Associated Vertebral Fractures After Denosumab Discontinuation: Systematic Review and Additional Cases.J Bone Miner Res, vol. 32 (6), 1291-1296. doi:10.1002/jbmr.3110. 